\title{
Current status of heart failure: global and Korea
}

\author{
Jin Joo Park and Dong-Ju Choi
}

Cardiovascular Center, Division of Cardiology, Seoul National University Bundang Hospital, Seongnam, Korea

Received: March 28, 2020

Accepted: April 23, 2020

\section{Correspondence to}

Dong-Ju Choi, M.D.

Cardiovascular Center, Division of Cardiology, Seoul National University Bundang Hospital, 82 Gumi-ro 173beon-gil, Bundanggu, Seongnam 13620, Korea

Tel: +82-31-787-7007

Fax: $+82-31-787-7041$

E-mail: djchoi@snubh.org https://orcid.org/0000-00030146-2189

This paper was contributed by The Korean Society of Heart Failure.
Heart failure (HF) is a condition in which the heart is unable to pump enough blood to meet the body's needs for blood and oxygen. Thus, HF is a grave disease with high morbidity and mortality. Because the prevalence of and exposure to the risk factors for HF increase with age, the prevalence of HF has been increasing in an aging society, including Korea. The vast advancement of medical and device therapy has improved the outcomes of HF, but significant residual risk still exists, and the benefit is confined to patients with reduced ejection fraction. Finding effective treatment for HF with preserved ejection fraction and identification of groups who benefit from drug and device therapy remain challenging. In this review, we illustrate the epidemiology, temporal trends, and current status of medical and device therapy, including heart transplantation, as well as emerging treatments for HF in Korea and worldwide.

Keywords: Heart failure; Epidemiology; Therapy; Korea; World

\section{INTRODUCTION}

Heart failure (HF) is a condition in which the heart is unable to pump enough blood to meet the body's needs for blood and oxygen [1]. It is a complex clinical syndrome characterized by definite symptoms (e.g., dyspnea or fatigue) and signs (e.g., increased jugular vein pressure, leg swelling, and pulmonary edema) caused by structural and/or functional abnormalities of the heart $[2,3]$. Hemodynamically, there is impairment of cardiac output and/or elevated intracardiac pressures at rest and during stress. It is important to note that $\mathrm{HF}$ is not synonymous with left ventricular dysfunction or cardiomyopathy alone; symptoms are necessary to diagnose HF.

\section{EPIDEMIOLOGY}

HF is a disease with high morbidity and mortality worldwide and in Korea. It affects more than 26 million people worldwide, and its prevalence is increasing [4]. The prevalence of HF varies between $0.1 \%$ and $6.7 \%$ worldwide.

Traditional risk factors for the development of HF include hypertension, diabetes mellitus, ischemic heart disease, and atrial fibrillation. Because the prevalence of and exposure to these risk factors increase with age, the prevalence of HF increases with the aging of the society. Indeed, in 1994, the prevalence of HF was 90 per 1,000 person-years and it increased to 121 per 1,000 person-years in 2003 among medicare beneficiaries 65 years or older in the United States [5]. Based on the National Health and Nutrition Examination Survey (NHANES) 2009 to 2012, an estimated 5.7 million adults had HF, whereas the NHANES 2013 to 2016 estimated that 6.2 million people $\geq 20$ years of age had HF in the United States. Projection analysis estimates that, in 2030, more than 8 million people in the United States 
$\geq 18$ years of age will have HF, which represents a $46 \%$ increase in prevalence from 2012 to 2030 . The total percentage of the population with HF is predicted to increase from $2.42 \%$ in 2012 to $2.97 \%$ in 2030 [6]. HF is the leading cause of hospitalization and is associated with enormous healthcare costs. More than 1 million people are hospitalized for HF each year, and the 1-year readmission rate is greater than $25 \%$ in the USA [7]. The total cost for HF was estimated to be $\$ 30.7$ billion in 2012 and is expected to increase by $127 \%$ to $\$ 69.8$ billion in 2030 [8]. Two-thirds of these costs are directly attributable to medical costs.

With a decrease in the birth rate and an increase in life expectancy, Korea became an aged society in 2017 , indicating that the elderly account for more than $14 \%$ of the total population. Consequently, the prevalence of $\mathrm{HF}$ is expected to rise owing to the change in population structure (Fig. 1A) [9]. In addition, the westernization of the Korean lifestyle and an increasing prevalence of ischemic heart disease also contribute to the rise in HF prevalence.

Using data from the 2002 to 2013 National Sample Cohort based on the National Health Information Database, the nationwide prevalence of HF in Korea was $0.75 \%$ in 2002 and $1.53 \%$ in 2012 (Fig. 1B). Interestingly, the projected HF prevalence is expected to be $1.89 \%$ and $3.35 \%$ in 2020 and 2040 , respectively. By 2040 , more than 1.7 million Koreans are expected to have $\mathrm{HF}[10]$.

The trend for increasing prevalence of HF is not a phenomenon confined to Korea but is observed worldwide in aging societies. Because the aging of the society appears to be inevitable, early and effective interventions to stop the progression of HF may be the only solution to prevent an HF epidemic [9].

\section{DEFINITION OF HF}

Left ventricular ejection fraction (LVEF) is an index of left ventricular contractility and is used to classify HF. The Korean Society of Heart Failure and the Heart Failure Association of the European Society of Cardiology define HF with normal LVEF ( $\geq 50 \%$ ) as HF with preserved ejection fraction ( $\mathrm{HFpEF}), \mathrm{HF}$ with decreased LVEF (< 40\%) as $\mathrm{HF}$ with reduced ejection fraction (HFrEF), and HF
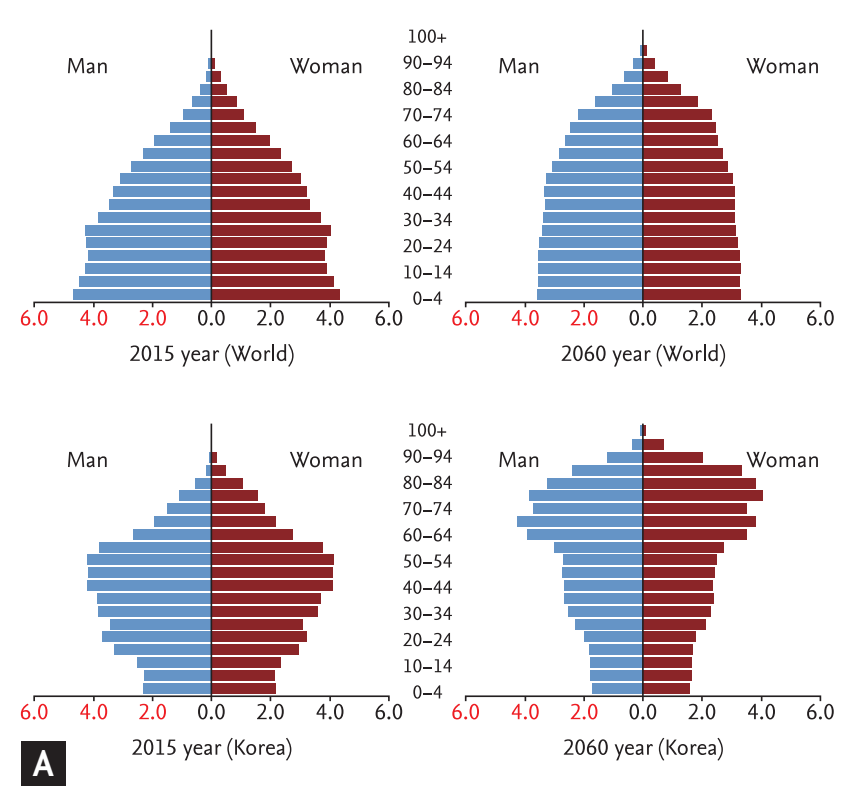

B

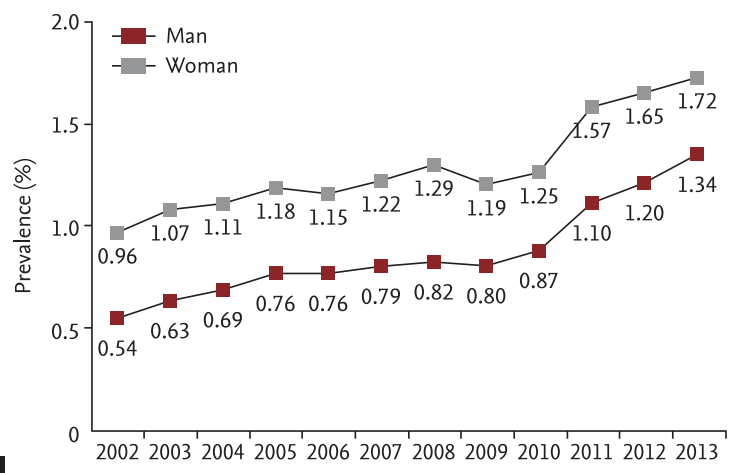

Year

Figure 1. Population structure in 2015 and expected population structure in Korea in 2060. (A) Population structure of the world in 2015 (upper left panel) and expected structure in 2060 (upper right panel); population structure of Korea in 2015 (lower left panel), and expected population structure in 2060 (lower right panel). Adapted from Park et al. [9]. (B) Prevalence of heart failure according to sex in 2002 to 2013 . Adapted from Lee et al. [10].

with LVEF ranging from $40 \%$ to $49 \%$ as $\mathrm{HF}$ with midrange ejection fraction (HFmrEF) [2,11]. By contrast, the American College of Cardiology Foundation/American Heart Association task force on practice guidelines categorize HF into HFrEF (LVEF < 40\%) and HFpEF (LVEF $\geq$ $40 \%$ ) groups only [3]. In the Korean Acute Heart Failure (KorAHF) registry, LVEF measurement was available in > 90\% patients: 3,088 (60.5\%) had LVEF that was $40 \%$ or less, while 1,285 (22.8\%) had LVEF greater than 50\% [12]. In the Korean Heart Failure (KorHF) registry, 743 (26.1\%) 
patients had LVEF > 50\% [13].

LVEF is not absolute, but it has relative value to volume status and is affected by heart rate in the acute setting. In addition, as the LVEF is not static, but changes during follow-up, some authors suggest reclassification of $\mathrm{HF}$ according to longitudinal LVEF changes. Patients with LVEF $<40 \%$ at baseline and $>40 \%$ at follow-up were defined as having HF with recovered ejection fraction (HFrecEF) or HF with improved ejection fraction (HFiEF) [14], whereas those with LVEF > 50\% at baseline and $<40 \%$ at follow-up were defined as having HF with a declined ejection fraction (HFdEF) [15].

\section{TEMPORAL TREND OF HF ACCORDING TO THE HF TYPE}

Using 15-year follow-up data from Olmsted County, Minnesota, from 1987 to 2001 , a similar trend of HF prevalence in the United States was illustrated. The proportion of patients with the diagnosis of $\mathrm{HFpEF}$ defined as LVEF > 50\% increased over time, whereas that of HFrEF did not change significantly over time [16]. Regarding survival, the patients with HFpEF had a slightly higher survival rate. Among patients with HFrEF, the likelihood of survival increased during the study period, reflecting the improvement of therapy in HFrEF, whereas that of HFpEF did not change significantly reflecting the lack of effective treatment in HF$\mathrm{pEF}[16]$.

\section{TEMPORAL TRENDS OF HF IN KOREA}

In Korea there are four representative HF registries spanning almost 4 decades starting from 1987 until 2011: (1) between January 1987 and December 1997: the Hallym HF study including 1,657 patients from four hospitals in a retrospective registry [17]; (2) between January 1998 and August 2003: the Korean Multicenter HF study including 1,759 patients with acute heart failure (AHF) in a nationwide, prospective registry from nine university hospitals [18]; (3) between June 2004 and April 2009 the KorHF registry including 3,200 patients with AHF in a nationwide prospective registry from 24 academic hospitals [13]; and (4) between March
2011 and March 2014: the KorAHF registry including 5,625 patients with AHF in a nationwide prospective, multicenter registry from 10 hospitals [12]. These four registries help us to understand the temporal changes in HF in Korea (Table 1) [12,13,17-25]. In the last four decades, there was an increase in the mean age of the patients from $63.7 \pm 13.4$ years in 1987 to $69 \pm 14$ years in 2011. The prevalence of hypertension, diabetes mellitus, and atrial fibrillation also increased over time. The predominant etiology of HF was ischemic cardiomyopathy owing to the westernization of lifestyle and comorbidities among Koreans and improved survival of patients with coronary artery disease, including those presenting with acute myocardial infarction who eventually progress to cardiac dysfunction. This trend is also seen in Japan [26] and China [10]. In contrast, HF due to valvular heart disease has been decreasing, reflecting the decreasing prevalence of valvular heart disease in Korea.

During hospitalization for AHF, more parenteral drugs and extracorporeal membrane oxygenation or percutaneous cardiopulmonary support were applied, and more patients underwent heart transplantation, which led to a marked improvement in in-hospital mortality, while the costs increased by about 40\% [12].

Regarding the post-discharge outcomes, the 1-year cumulative survival rate was $82 \%, 80.1 \%, 85 \%$, and 81.8\% for Hallym, Korea Multicenter, KorHF, and KorAHF, respectively. It is of note that direct comparison of the post-discharge outcomes is difficult because KorAHF validated survival using the National Insurance data or National Death Records mortality data, while the mortality was not validated in other registries, leaving the possibility of underestimation of the mortality rate. Taken together, the improvement in acute clinical care may have led to improved in-hospital outcomes, whereas long-term mortality remains unaffected.

\section{MEDICAL THERAPY}

Drugs targeting the sympathetic nervous system and neurohumoral activation have improved survival in patients with HFrEF [27]. To be more specific, angiotensin converting enzyme-inhibitors (ACEi), angio- 


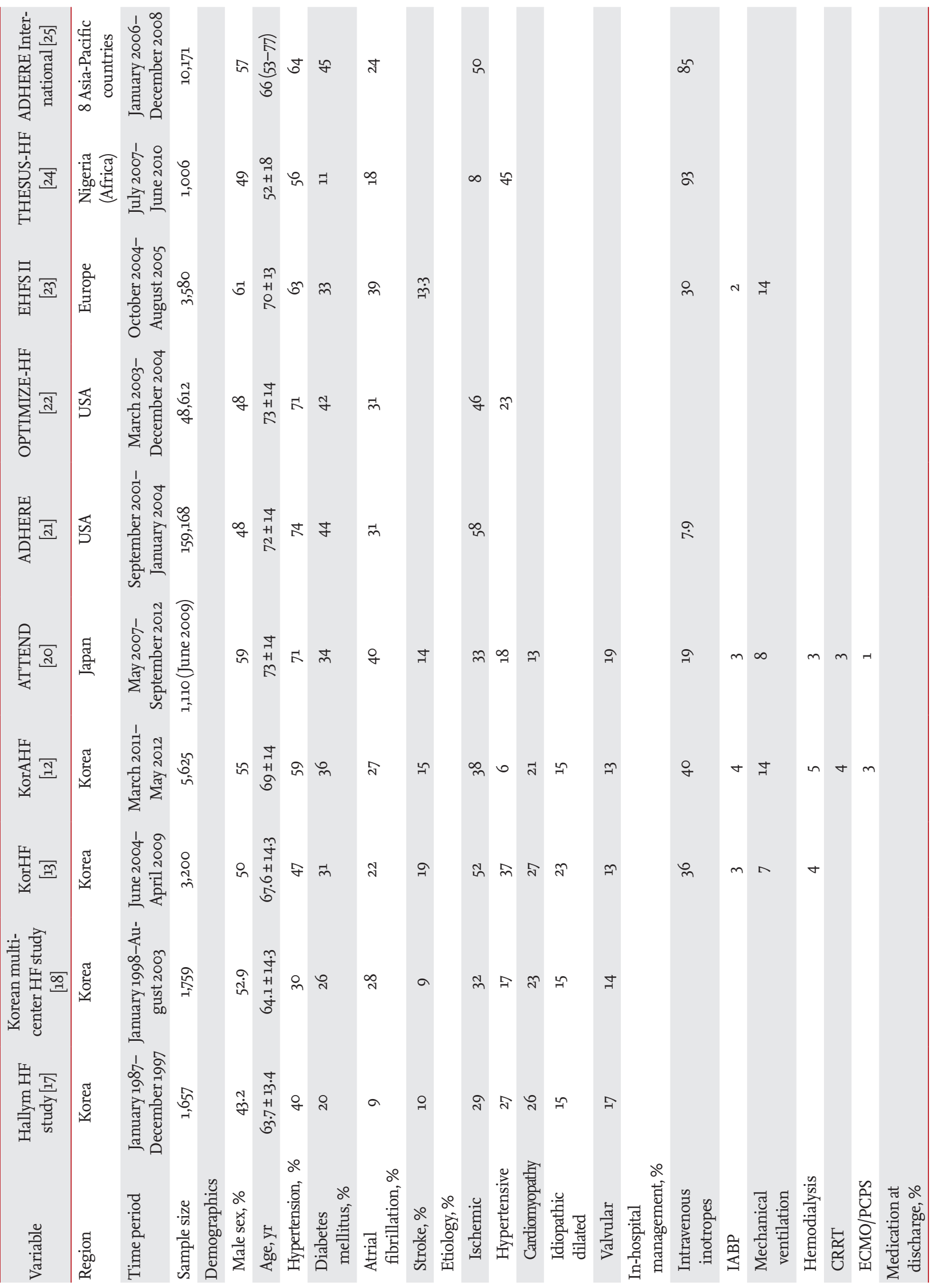




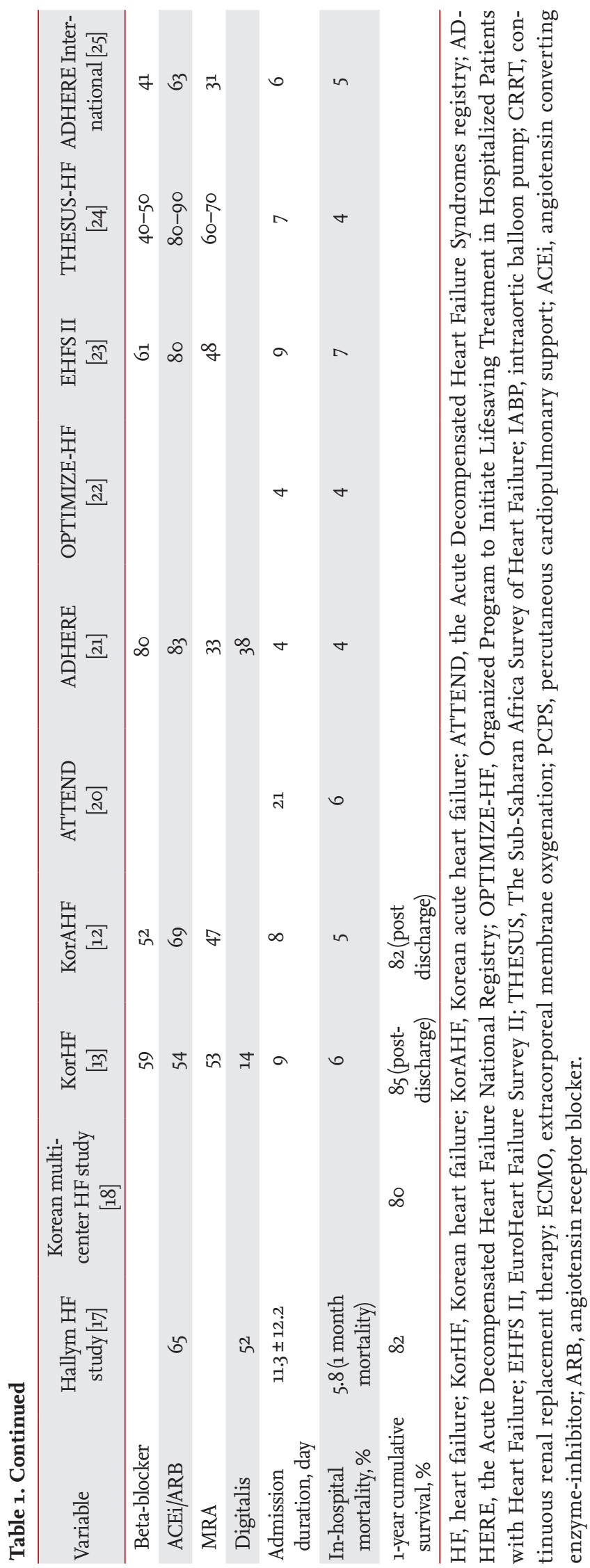

tenisn receptor blockers (ARBs), beta-blockers, mineralocorticoid receptor antagnoists (MRA), ivabradine, and recently sacubitril/valsartan were able to improve the clinical outcomes in patients with HFrEF [28-35]. In contrast, none of these drugs were able to improve survival in patients with HFpEF [36-39].

The KorAHF-Registry provides an important insight into drug prescription patterns during AHF admission. The prescription rate of the drugs was generally low at admission, which may be explained by the fact that $52 \%$ of the patients had de novo (new-onset) HF and were possibly drug naïve. The prescription rates reached a peak during hospitalization but declined by discharge, inferring that physicians attempt to initiate guideline-directed medical therapy but must discontinue some drugs due to intolerability. At discharge from AHF admission, 68.8\%, 52.2\%, and 46.6\% of patients were on renin-angiotensin-system inhibitors (RAS inhibitors, ACEi, or ARB), beta-blockers, or MRAs, respectively (Fig. 2). When stratifying the patients according to the indications of each drug, that is, RAS inhibitors and beta-blockers in patients with LVEF $\leq 40 \%$ and MRAs in patients with LVEF $\leq 35 \%$, $77.6 \%, 58.0 \%$, and $55.1 \%$ of the patients were treated with RAS inhibitors, beta-blockers, or MRAs at discharge, respectively.

Many patients with HFrEF experience improvement or recovery of LVEF. In the KorAHF registry, 2,302 (75\%) patients with HFrEF had baseline and follow-up echocardiography at 12 months. HF phenotypes were defined as persistent HFrEF (LVEF $\leq 40 \%$ at baseline and at the 1-year follow-up) or HFiEF (LVEF $\leq 40 \%$ at baseline and improved to $>40 \%$ at 1-year follow-up), and those with HFiEF had a lower mortality rate compared to patients with persistent HFrEF [14]. Regarding medical treatment, beta-blockers, but not RAS inhibitors or MRAs, were associated with a reduced all-cause mortality risk. The benefits seemed similar in patients receiving low- or high-dose beta-blockers. The study implies that although HFiEF is a distinct HF phenotype with better clinical outcomes than those of other phenotypes, beta-blockers should be continued in these patients [28].

Despite progress in HF therapies and improvement of the prognosis, there is still a substantial residual risk for death, stimulating the search for new therapeutic 


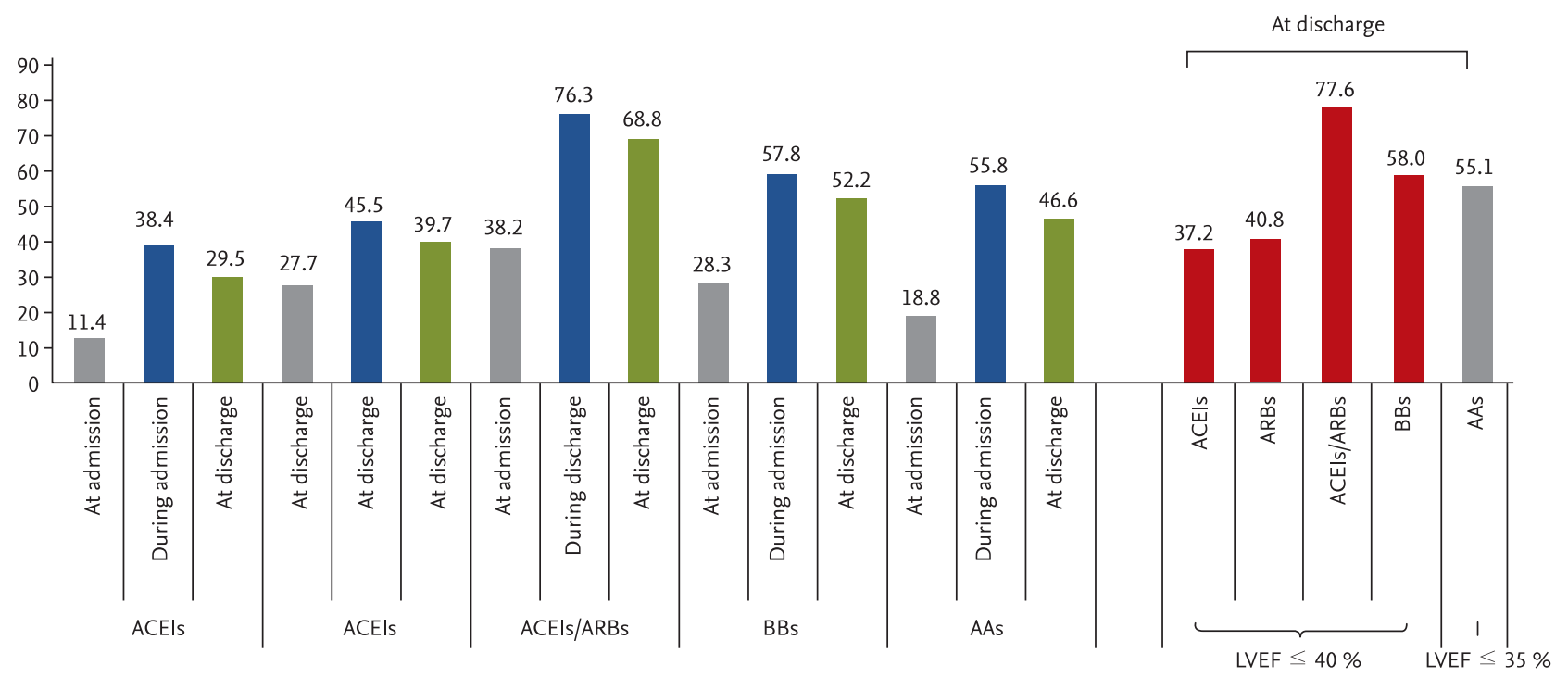

Figure 2. Evidence-based medication prescriptions. Adapted from Lee et al. [12]. ACEI, angiotensin converting enzyme-inhibitor; ARB, angiotensin receptor blocker; BB, beta-blocker; AA, aldosterone antagonist; LVEF, left ventricular ejection fraction.

options. Diabetes is an important risk factor for HF. Although an increase in hemoglobin Alc is associated with an increased risk of $\mathrm{HF}$, intensive glycemic control does not reduce the incidence of $\mathrm{HF}[40,41]$.

Because of the potential risk of adverse cardiovascular outcomes of antidiabetic drugs, they are required to undergo cardiovascular outcome trials to prove their safety. Sodium-glucose transporter 2 (SGLT2) inhibitors are a new class of oral antidiabetic drugs. In cardiovascular outcome trials (Table 2), empagliflozin [42], canagliflozin [43], and dapagliflozin [44] showed a robust reduction in hospitalization for HF in patients with type 2 diabetes mellitus with and without established atherosclerotic cardiovascular disease (ASCVD) [45]. This benefit was observed in a broad population regardless of prior HF, established ASCVD, ejection fraction, or kidney function [46].

The Study to Evaluate the Effect of Dapagliflozin on the Incidence of Worsening Heart Failure or Cardiovascular Death in Patients With Chronic Heart Failure With Reduced Ejection Fraction (DAPA-HF) trial [47] investigated the effect of dapagliflozin in patients with HFrEF with and without diabetes who received optimal medical treatment including beta-blockers, RAS inhibitors, sacubitril/valsartan, and MRAs. Dapagliflozin reduced the composite of $\mathrm{CV}$ death, hospitalization for HF, and urgent HF visits by $26 \%$. Thus, SGLT2 in- hibitors seem to be emerging drugs for patients with HFrEF.

In the withdrawal of pharmacological treatment for HF in patients with recovered dilated cardiomyopathy (TRED-HF) study, 51 patients with previous dilated cardiomyopathy whose LVEF had improved from < $40 \%$ to $\geq 50 \%$, whose left ventricular end-diastolic volume (LVEDV) had normalized, and who had an N-terminal pro-B-type natriuretic peptide concentration $<250 \mathrm{ng} / \mathrm{L}$ were randomly assigned to phased withdrawal or continuation of treatment [48]. Over the first 6 months, $44 \%$ of the patients randomly assigned to treatment withdrawal experienced a relapse compared with none of those assigned to continue treatment. The study implies that many patients who recover from dilated cardiomyopathy will relapse following treatment withdrawal and suggests continuing treatment indefinitely.

On the contrary, some patients with HFpEF experienced a decline in LVEF during follow-up. Of the patients with HFpEF in the KorAHF registry, 426 (90.4\%) were diagnosed as having persistent $\mathrm{HFpEF}$ and 45 (9.6\%) as having HFdEF defined as LVEF $\geq 50 \%$ at admission and $<50 \%$ at 1 year [15]. Patients with HFdEF had a worse prognosis, and the use of beta-blockers, RAS inhibitors, and MRAs was not associated with improved prognosis of patients with HFdEF. 


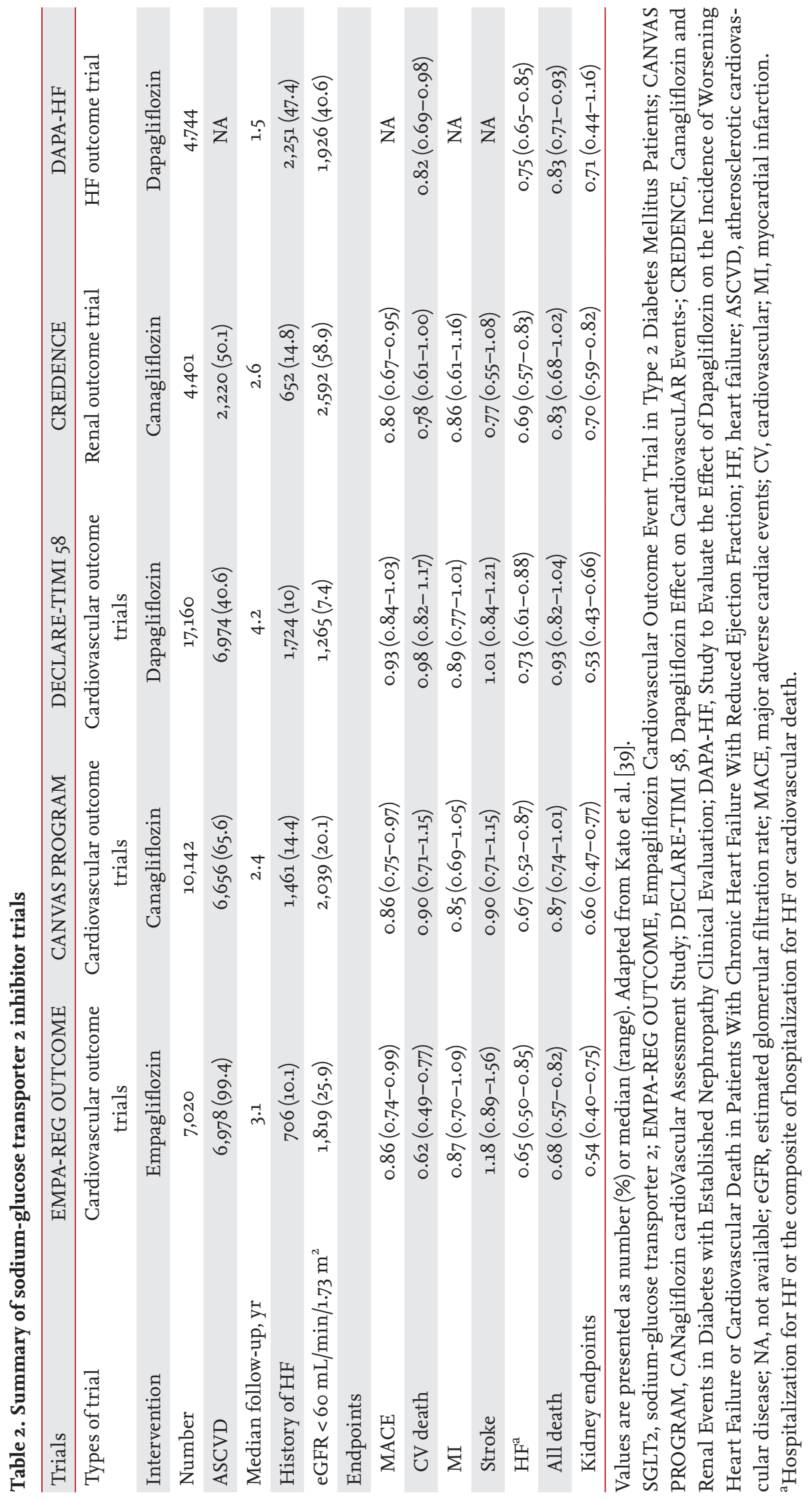



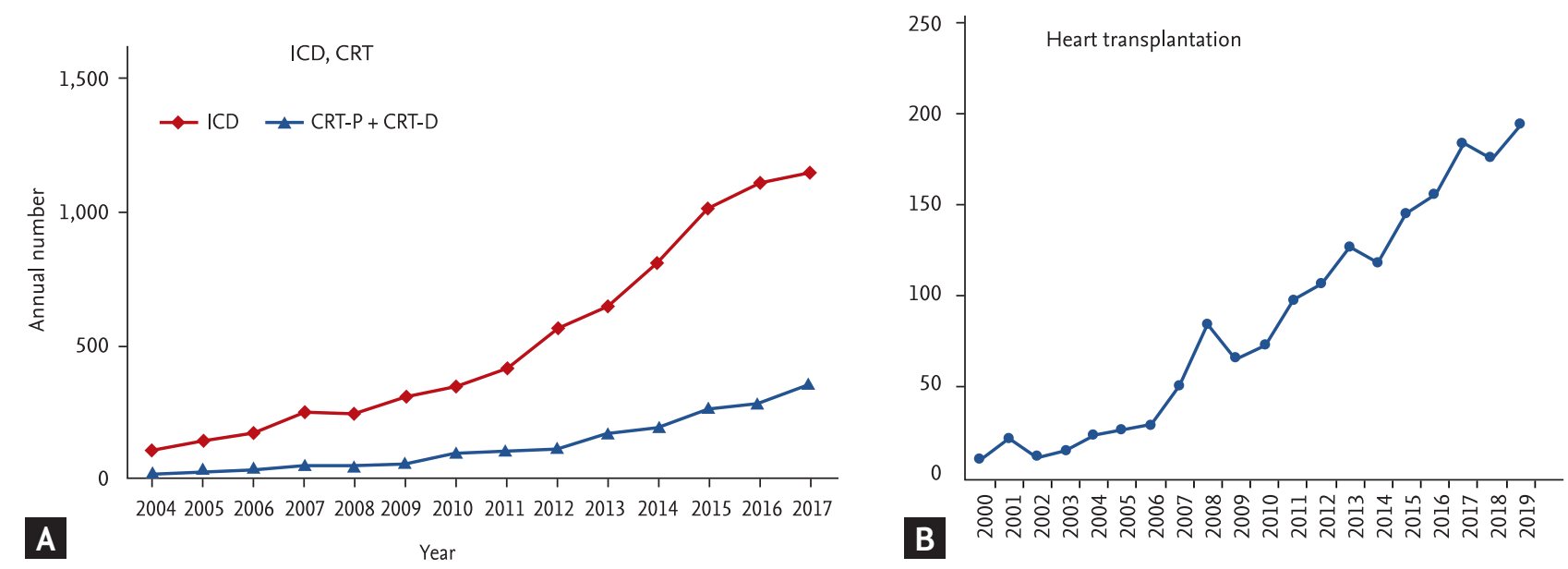

Figure 3. Temporal trends of device therapy and heart transplantation. (A) Cardiac implantable electronic device implantation in Korea. (B) Heart transplantation. Adapted from Choi et al. [52]. ICD, implantable cardioverter-defibrillator; CRT-P, cardiac resynchronization therapy without defibrillator; CRT-D, cardiac resynchronization therapy with defibrillator.

\section{DEVICE THERAPY AND HEART TRANSPLANTATION}

The current guidelines recommend an implantable cardioverter defibrillator (ICD) for primary prevention in symptomatic HF patients with $\mathrm{LVEF} \leq 35 \%$ despite $>3$ months of optimal medical therapy $[2,11]$. Cardiac resynchronization therapy (CRT) is indicated in symptomatic patients with $\mathrm{LVEF} \leq 35 \%$ despite optimal medical therapy who have a left bundle branch block and a QRS duration $\geq 130 \mathrm{~ms}[2,11]$. There has been an underutilization of ICD and CRT in Korea. In the United States, the ICD implantation rate was 46.2 cases per 100,000 persons in 2006 [49], whereas in Western Europe, the CRT device implantation rate was 19.7 per 100,000 persons in 2014 [50]. Furthermore, the ICD implantation rate in Japan was seven times (o.6 vs. 4.2 in 2009) higher than that of Korea from 2009 to 2010 [51]. However, with reimbursement for the device and increasing awareness and education of HF patients and physicians, there was has been a steady increase in device therapy in patients with $\mathrm{HF}$ (Fig. 3A) [52].

Because of the improvements in the treatment of $\mathrm{HF}$, an increasing number of patients are living with advanced-stage HF [53]. Heart transplantation is a valid treatment option for patients with end-stage HF who are deemed to be appropriate candidates. Improvements in immunosuppressants, donor procurement, surgical techniques, and post-transplant care have resulted in a substantial decrease in allograft rejection and increased survival, exercise capacity, and quality of life $[54,55]$. In Korea, the first heart transplantation was performed in 1992, and by the end of 2019, over 1700 patients had undergone heart transplantation (Fig. 3B). Because the number of patients with end-stage HF who are eligible for heart transplantation is increasing, there is a significant organ shortage and a long waiting time. The disparity between available donor hearts and cardiac transplant candidates led to the development of mechanical circulatory support (MCS) devices, and durable and implantable MCS devices are used as a bridge to cardiac transplantation. Since 2019, left ventricular assist devices have been reimbursed by the Korean National Health Insurance and have been actively used as a bridge to cardiac transplantation in patients requiring MCS in Korea.

\section{HEART FAILURE WITH PRESERVED EJECTION FRACTION}

Although patients with HFpEF have normal or preserved LVEF, they have a prognosis similar to HFrEF. HFpEF is challenging because the underlying pathophysiology is poorly understood; therefore, there are controversies regarding the diagnostic criteria, and its diagnosis can be difficult even for HF specialists [56]. More importantly, until now, no therapy has been shown to improve the outcomes in these patients. 
In the Japanese Diastolic Heart Failure (J-DHF) study with 245 patients with LVEF > 40\%, carvedilol did not improve the prognosis of patients with HFpEF [37]. In an individual patient-level meta-analysis of 11 trials, Cleland et al. [57] also demonstrated that administration of beta-blockers did not improve outcomes in patients with HFpEF defined as an LVEF of $\geq 50 \%$. Regarding RAS inhibitors, perindopril [58], irbesartan [36], candesartan [59], and spironolactone [38] showed neutral results in terms of survival gain. Similarly, sacubitril/valsartan, an angiotensin-receptor-neprilysin inhibitor, failed to improve survival in patients with HFpEF [39]. Thus, it is of clinical interest and importance to determine whether SGLT2 inhibitors can improve the outcomes in patients with HFpEF and change the practice guidelines.

\section{CONCLUSIONS}

HF is a disease with high morbidity and mortality. With an aging society, its prevalence is expected to grow in Korea and worldwide. Although there have been enormous advancements in treatment that have significantly improved the outcomes of HF, significant residual risk exists, and the benefit has been confined to HFrEF. Finding effective treatment for HFpEF and identifying groups of patients who would benefit from drug and device therapy remains a challenge.

\section{Conflict of interest}

No potential conflict of interest relevant to this article was reported.

\section{REFERENCES}

1. Coronel R, de Groot JR, van Lieshout JJ. Defining heart failure. Cardiovasc Res 2001;50:419-422.

2. Ponikowski P, Voors AA, Anker SD, et al. 2016 ESC guidelines for the diagnosis and treatment of acute and chronic heart failure: the task force for the diagnosis and treatment of acute and chronic heart failure of the European Society of Cardiology (ESC). Developed with the special contribution of the Heart Failure Association (HFA) of the ESC. Eur J Heart Fail 2016;18:891-975.

3. Yancy CW, Jessup M, Bozkurt B, et al. 2013 ACCF/AHA guideline for the management of heart failure: a report of the American College of Cardiology Foundation/American Heart Association Task Force on Practice Guidelines. J Am Coll Cardiol 2013;62:e147-e239.

4. Savarese G, Lund LH. Global public health burden of heart failure. Card Fail Rev 2017;3:7-11.

5. Curtis LH, Whellan DJ, Hammill BG, et al. Incidence and prevalence of heart failure in elderly persons, 1994-2003. Arch Intern Med 2008;168:418-424.

6. Virani SS, Alonso A, Benjamin EJ, et al. Heart disease and stroke statistics-2020 update: a report from the American Heart Association. Circulation 2020;141:e139-e596.

7. Dharmarajan K, Hsieh AF, Lin Z, et al. Diagnoses and timing of 30-day readmissions after hospitalization for heart failure, acute myocardial infarction, or pneumonia. JAMA 2013;309:355-363.

8. Mora S, Wenger NK, Cook NR, et al. Evaluation of the pooled cohort risk equations for cardiovascular risk prediction in a multiethnic cohort from the Women's Health Initiative. JAMA Intern Med 2018;178:1231-1240.

9. Park JJ, Choi DJ. Heart failure epidemic: an expected phenomenon. Korean Circ J 2016;46:610-612.

10. Lee JH, Lim NK, Cho MC, Park HY. Epidemiology of heart failure in Korea: present and future. Korean Circ J 2016;46:658-664.

11. Kim KJ, Cho HJ, Kim MS, et al. Focused update of 2016 Korean Society of Heart Failure guidelines for the management of chronic heart failure. Int J Heart Fail 2019;1:424.

12. Lee SE, Lee HY, Cho HJ, et al. Clinical characteristics and outcome of acute heart failure in Korea: results from the Korean Acute Heart Failure Registry (KorAHF). Korean Circ J 2017;47:341-353.

13. Choi DJ, Han S, Jeon ES, et al. Characteristics, outcomes and predictors of long-term mortality for patients hospitalized for acute heart failure: a report from the Korean Heart Failure Registry. Korean Circ J 2011;41:363-371.

14. Park CS, Park JJ, Mebazaa A, et al. Characteristics, outcomes, and treatment of heart failure with improved ejection fraction. J Am Heart Assoc 2019;8:e011077.

15. Park JJ, Park CS, Mebazaa A, et al. Characteristics and outcomes of HFpEF with declining ejection fraction. Clin Res Cardiol 2020;109:225-234.

16. Owan TE, Hodge DO, Herges RM, Jacobsen SJ, Roger VL, Redfield MM. Trends in prevalence and outcome of heart failure with preserved ejection fraction. N Engl J Med 
2006;355:251-259.

17. Lee Y. Clinical characteristics of Korean patients with congestive heart failure. Korean J Med 1998;55:446-452.

18. Han SW, Ryu KH, Chae SC, et al. Multicenter analysis of clinical characteristics and prognostic factors of patients with congestive heart failure in Korea. Korean Circ J 2005;35:357-361.

19. Youn JC, Han S, Ryu KH. Temporal trends of hospitalized patients with heart failure in Korea. Korean Circ J 2017;47:16-24.

20. Sato N, Kajimoto K, Keida T, et al. Clinical features and outcome in hospitalized heart failure in Japan (from the ATTEND Registry). Circ J 2013;77:944-951.

21. Fonarow GC, Heywood JT, Heidenreich PA, Lopatin M, Yancy CW; ADHERE Scientific Advisory Committee and Investigators. Temporal trends in clinical characteristics, treatments, and outcomes for heart failure hospitalizations, 2002 to 2004: findings from Acute Decompensated Heart Failure National Registry (ADHERE). Am Heart J 2007;153:1021-1028.

22. Abraham WT, Fonarow GC, Albert NM, et al. Predictors of in-hospital mortality in patients hospitalized for heart failure: insights from the Organized Program to Initiate Lifesaving Treatment in Hospitalized Patients with Heart Failure (OPTIMIZE-HF). J Am Coll Cardiol 2008;52:347356.

23. Nieminen MS, Brutsaert D, Dickstein K, et al. EuroHeart Failure Survey II (EHFS II): a survey on hospitalized acute heart failure patients: description of population. Eur Heart J 2006;27:2725-2736.

24. Damasceno A, Mayosi BM, Sani M, et al. The causes, treatment, and outcome of acute heart failure in 1006 Africans from 9 countries. Arch Intern Med 2012;172:1386-1394.

25. West R, Liang L, Fonarow GC, et al. Characterization of heart failure patients with preserved ejection fraction: a comparison between ADHERE-US registry and ADHERE-International registry. Eur J Heart Fail 2011;13:945952.

26. Okura Y, Ramadan MM, Ohno Y, et al. Impending epidemic: future projection of heart failure in Japan to the year 2055. Circ J 2008;72:489-491.

27. Lee HY, Oh BH. Paradigm shifts of heart failure therapy: do we need another paradigm? Int J Heart Fail. 2020;2:e3.

28. Packer M, Bristow MR, Cohn JN, et al. The effect of carvedilol on morbidity and mortality in patients with chronic heart failure. U.S. Carvedilol Heart Failure Study Group.
N Engl J Med 1996;334:1349-1355.

29. CIBIS-II Investigators and Committees. The Cardiac Insufficiency Bisoprolol Study II (CIBIS-II): a randomized trial. Lancet 1999;353:9-13.

30. SOLVD Investigators, Yusuf S, Pitt B, Davis CE, Hood WB, Cohn JN. Effect of enalapril on survival in patients with reduced left ventricular ejection fractions and congestive heart failure. N Engl J Med 1991;325:293-302.

31. Pitt B, Zannad F, Remme WJ, et al. The effect of spironolactone on morbidity and mortality in patients with severe heart failure. Randomized Aldactone Evaluation Study Investigators. N Engl J Med 1999;341:709-717.

32. Cohn JN, Tognoni G; Valsartan Heart Failure Trial Investigators. A randomized trial of the angiotensin-receptor blocker valsartan in chronic heart failure. N Engl J Med 2001;345:1667-1675.

33. Swedberg K, Komajda M, Bohm M, et al. Ivabradine and outcomes in chronic heart failure (SHIFT): a randomised placebo-controlled study. Lancet 2010;376:875-885.

34. McMurray JJ, Packer M, Desai AS, et al. Angiotensin-neprilysin inhibition versus enalapril in heart failure. N Engl J Med 2014;371:993-1004.

35. Greenberg B. Angiotensin receptor-neprilysin inhibition (ARNI) in heart failure. Int J Heart Fail 2020;2:73-90.

36. Massie BM, Carson PE, McMurray JJ, et al. Irbesartan in patients with heart failure and preserved ejection fraction. N Engl J Med 2008;359:2456-2467.

37. Yamamoto K, Origasa H, Hori M; J-DHF Investigators. Effects of carvedilol on heart failure with preserved ejection fraction: the Japanese Diastolic Heart Failure Study (J-DHF). Eur J Heart Fail 2013;15:110-118.

38. Pitt B, Pfeffer MA, Assmann SF, et al. Spironolactone for heart failure with preserved ejection fraction. N Engl J Med 2014;370:1383-1392.

39. Solomon SD, McMurray JJV, Anand IS, et al. Angiotensin-neprilysin inhibition in heart failure with preserved ejection fraction. N Engl J Med 2019;381:1609-1620.

40. Iribarren C, Karter AJ, Go AS, et al. Glycemic control and heart failure among adult patients with diabetes. Circulation 2001;103:2668-2673.

41. Stratton IM, Adler AI, Neil HA, et al. Association of glycaemia with macrovascular and microvascular complications of type 2 diabetes (UKPDS 35): prospective observational study. BMJ 2000;321:405-412.

42. Zinman B, Wanner C, Lachin JM, et al. Empagliflozin, cardiovascular outcomes, and mortality in type 2 diabetes. 
N Engl J Med 2015;373:2117-2128.

43. Neal B, Perkovic V, Mahaffey KW, et al. Canagliflozin and cardiovascular and renal events in type 2 diabetes. $\mathrm{N}$ Engl J Med 2017;377:644-657.

44. Wiviott SD, Raz I, Bonaca MP, et al. Dapagliflozin and cardiovascular outcomes in type 2 diabetes. $\mathrm{N}$ Engl J Med 2019;380:347-357.

45. Kato ET, Kimura T. Sodium-glucose co-transporters-2 inhibitors and heart failure: state of the art review and future potentials. Int J Heart Fail 2020;2:12-22.

46. Zelniker TA, Wiviott SD, Raz I, et al. SGLT2 inhibitors for primary and secondary prevention of cardiovascular and renal outcomes in type 2 diabetes: a systematic review and meta-analysis of cardiovascular outcome trials. Lancet 2019;393:31-39.

47. McMurray JJV, Solomon SD, Inzucchi SE, et al. Dapagliflozin in patients with heart failure and reduced ejection fraction. N Engl J Med 2019;381:1995-2008.

48. Halliday BP, Wassall R, Lota AS, et al. Withdrawal of pharmacological treatment for heart failure in patients with recovered dilated cardiomyopathy (TRED-HF): an open-label, pilot, randomised trial. Lancet 2019;393:61-73.

49. Greenspon AJ, Patel JD, Lau E, et al. 16-Year trends in the infection burden for pacemakers and implantable cardioverter-defibrillators in the United States 1993 to 2008. J Am Coll Cardiol 2011;58:1001-1006.

50. Raatikainen MJ, Arnar DO, Zeppenfeld K, Merino JL, Kuck KH, Hindricks G. Current trends in the use of cardiac implantable electronic devices and interventional electrophysiological procedures in the European Society of Cardiology member countries: 2015 report from the European Heart Rhythm Association. Europace 2015;17:iv1-iv72.

51. Lee JH, Lee SR, Choi EK, et al. Temporal trends of cardiac implantable electronic device implantations: a nationwide population-based study. Korean Circ J 2019;49:841852.

52. Choi HM, Park MS, Youn JC. Update on heart failure management and future directions. Korean J Intern Med 2019;34:11-43.

53. Holley CT, Harvey L, John R. Left ventricular assist devices as a bridge to cardiac transplantation. J Thorac Dis 2014;6:1110-1119.

54. Banner NR, Bonser RS, Clark AL, et al. UK guidelines for referral and assessment of adults for heart transplantation. Heart 2011;97:1520-1527.

55. Mehra MR, Kobashigawa J, Starling R, et al. Listing criteria for heart transplantation: International Society for Heart and Lung Transplantation guidelines for the care of cardiac transplant candidates: 2006. J Heart Lung Transplant 2006;25:1024-1042.

56. Choi DJ, Park JJ, Ali T, Lee S. Artificial intelligence for the diagnosis of heart failure. NPJ Digit Med 2020;3:54.

57. Cleland JGF, Bunting KV, Flather MD, et al. Beta-blockers for heart failure with reduced, mid-range, and preserved ejection fraction: an individual patient-level analysis of double-blind randomized trials. Eur Heart J 2018;39:26-35.

58. Cleland JG, Tendera M, Adamus J, et al. The perindopril in elderly people with chronic heart failure (PEP-CHF) study. Eur Heart J 2006;27:2338-2345.

59. Yusuf S, Pfeffer MA, Swedberg K, et al. Effects of candesartan in patients with chronic heart failure and preserved left-ventricular ejection fraction: the CHARM-Preserved Trial. Lancet 2003;362:777-781. 